\title{
Railway overhead contact wire monitoring system by means of FBG sensors
}

\author{
Carla Lupi, Ferdinando Felli, Erwin Ciro \\ DICMA, Sapienza Università di Roma, Via Eudossiana 18, 00184 Roma, Italy \\ carla.lupi@uniroma1.it, bttp:/ /orcid.org/0000-0003-4096-3592 \\ ferdinando.felli@uniroma1.it, bttps:/ / orcid.org/0000-0002-7527-8096 \\ erwin.ciro@uniroma1.it, bttps://orcid.org/0000-0002-7673-3556
}

\section{Claudio Paris}

Centro Ricerche Enrico Fermi, Via Panisperna 89a, 00184 Roma, Italy

claudio.paris@centrofermi.it, https://orcid.org/0000-0003-3137-1456

\section{Cristian Vendittozzi}

Faculty UnB Gama, Universidade de Brasilia - UnB, Brasilia, 72444-240 Gama Brasilia-DF, Brazil vendittozzi@unb.brhttps:/ /orcid.org/0000-0003-0793-4052

\begin{abstract}
Safety of infrastructures represents one of the most significant concerns for governments and service providers to preserve people's wellbeing. One of the main ways to keep safe facilities (buildings, bridges, railways, etc.) involves the use of monitoring sensor systems in charge of measuring critical operating conditions. Those measurements together with periodical maintenance, contribute to minimize potential risks that the infrastructure faces. The paper aims at designing, developing, and testing a monitoring system for mechanical stresses acting on the overhead contact wire (OCW) to ensure the operational safety of the railway network. In this regard, the paper proposes two Fiber Bragg Grating (FBG) sensors-based solutions, relying on the ability of these sensors to allow real-time and continuous data acquisition. The first one consists of a polyimide-coated sensor bonded on an OCW clamp, the second one is a copper-coated sensor hanging between the two separated halves of an OCW clamp. Significant results have been obtained mechanically testing both solutions, trying to simulate the operative conditions.
\end{abstract}

KEYwORDS. FBG; OCW; Embedded Sensory System; Electrodeposited metal coating; Strain measuring.

\section{OPEN ACCESS}

Citation: Lupi, C., Felli, F., Ciro, E., Paris, C., Vendittozzi, C., Railway overhead contact wire monitoring system by means of FBG sensors, Frattura ed Integrità Strutturale, 57 (2021) 246-258.

Received: 14.05 .2021

Accepted: 10.06 .2021

Published: 01.07.2021

Copyright: (c) 2020 This is an open access article under the terms of the CC-BY 4.0, which permits unrestricted use, distribution, and reproduction in any medium, provided the original author and source are credited. 


\section{INTRODUCTION}

I $\mathrm{n}$ the railway system several components undergo constant monitoring and frequent maintenance due to severe working and environmental conditions. Particularly, the rails and the overhead contact wires (OCW) require permanent monitoring. In the current paper a system for monitoring the $\mathrm{OCW}$ is presented. The most common configuration involved in overhead contact system (OCS) comprises a complex electric structure consisting of tension-carrying wires to provide power transmission to trains. Some of these cable wires are OCW and messenger wire. These two wires are longitudinally supported by a cantilever array describing a zig-zag distribution along the electric system (overhead catenary). Both the $\mathrm{OCW}$ and messenger wire are distanced by dropper cables responsible to minimize falls and maintain the vertical elasticity [1-3], (Fig. 1).

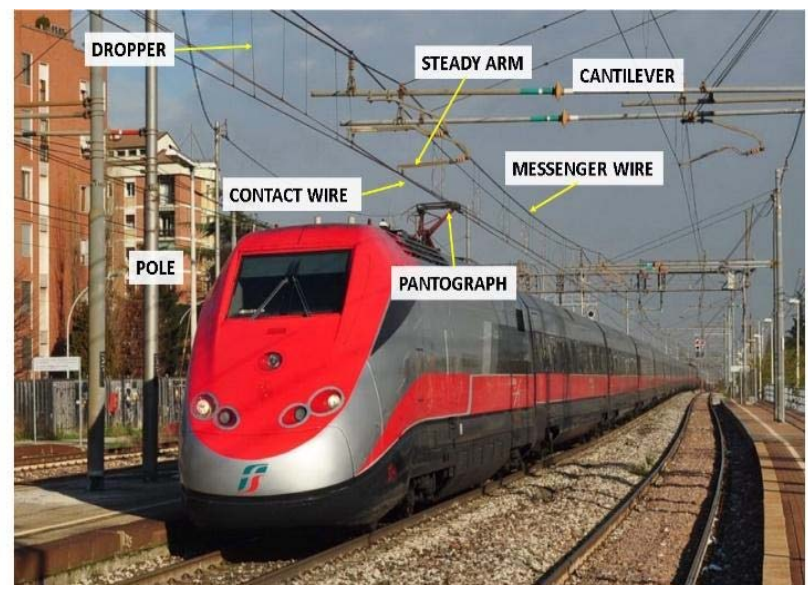

Figure 1: Overhead contact system (also known as catenary) on an Italian railway.

The most important problems regarding OCW can be normally summarized in contact loss and excessive wear with pantographs. The continuous friction between the pantograph and the OCW produces wear that reduces the effective cross section of the wire (Fig. 2).

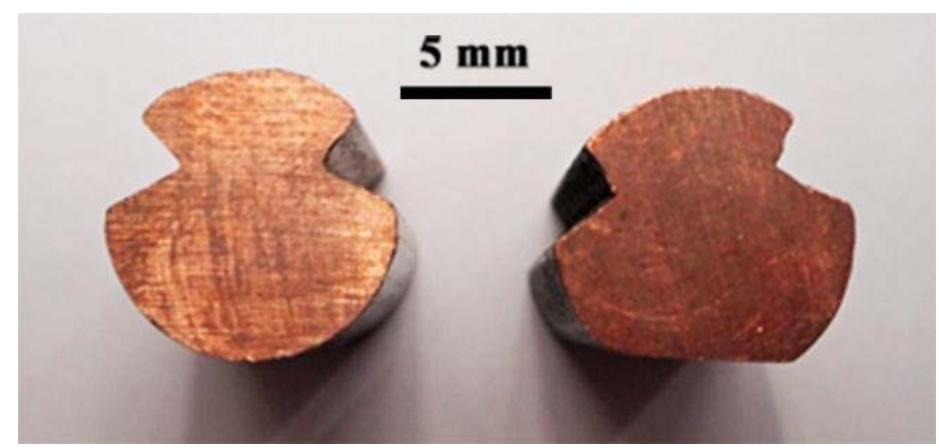

Figure 2: OCW cross section detail showing the wire before (on the left) and after (on the right) wear action.

The excessive wear issue often leads to energy interruptions and consequently to local arcing formation once operations restart. These conditions result in an undesirable combination of electrical and mechanical effects causing the performance deterioration of both systems, overcharges, and an inability to provide proper service. Although both the contact and the energy collection capabilities might be enhanced, it is also true that both systems suffer rapid wear. Several studies have been carried out to understand and measure the wear mechanism [1-4], and to develop new chemical compositions of OCW, shifting from pure copper to copper-based carbon composites [5-7]. In order to ensure railway service quality and safety, $\mathrm{OCW}$ wear measurements are required. Thus, several investigations are aimed at designing an automatic monitoring system for detecting early-stage deterioration of $\mathrm{OCW}$, resulting in excessive wear, through continuous and in real-time monitoring, of the mechanical and thermal load variations acting on the OCW. In this context different contactless measurement 
techniques have been investigated (based on magnetic fields analysis, microwave reflection analysis, electrical and optical detection systems) for solving the wear issues [7-10].

This investigation seeks to monitor the mechanical stresses that deteriorate OCWs by means of an easy-to-install smart clamp (SC) provided by a copper-coated sensor. This paper neglects the effect of temperature, which is another fundamental parameter that affects the OCW's stress state (wire tensions vary somewhat unpredictably with temperature variations). This is because temperature has a critical effect on the operative performance of the railway network when exceeding the thermal ranges $\left(-40^{\circ} \mathrm{C}\right.$ and $\left.60^{\circ} \mathrm{C}\right)[11,12]$. The study of the interaction between the $\mathrm{OCW}$ and its thermal field will be the focus of forthcoming investigations.

In unstressed conditions with no passing trains, an increase of stress on the contact wire means a wire-section reduction. If a cross-section reaches a reduction of $20 \%$, the wire should be replaced in compliance with railway regulations, [13]. In the present paper all references to railway nomenclature and regulations refer to the Italian railway network (RFI, that stands for Rete Ferroviaria Italiana).

To evaluate mechanical stresses of the OCW, two smart measuring systems were developed integrating Fiber Bragg Grating (FBG) sensors into standard railways clamps. Previous laboratory observations suggest that directly bonding the sensors on the contact wire can cause their failure, mainly because of its intrinsic brittleness. Some critical issues of FBG sensors bonded to the OCW could be: i) measurements of temperature and strain are unreliable when the collector plate of the pantograph passes close to the sensor, ii) thermal compensation in the sensor strain measurements can be difficult to be achieved, iii) preservation of sensor-system from environmental effects, iv) no possibility to remove the FGB from OCW, if required.

The vision is that of an intelligent system capable of monitoring the OCW continuously and in real time, consisting of an array of sensors that can be easily integrated into existing systems on the national railway network and perfectly compliant with standard operating conditions. The whole monitoring system includes a control room from which several sensors chains branch out, covering railway stretches of tens of km, as shown in Fig. 3.

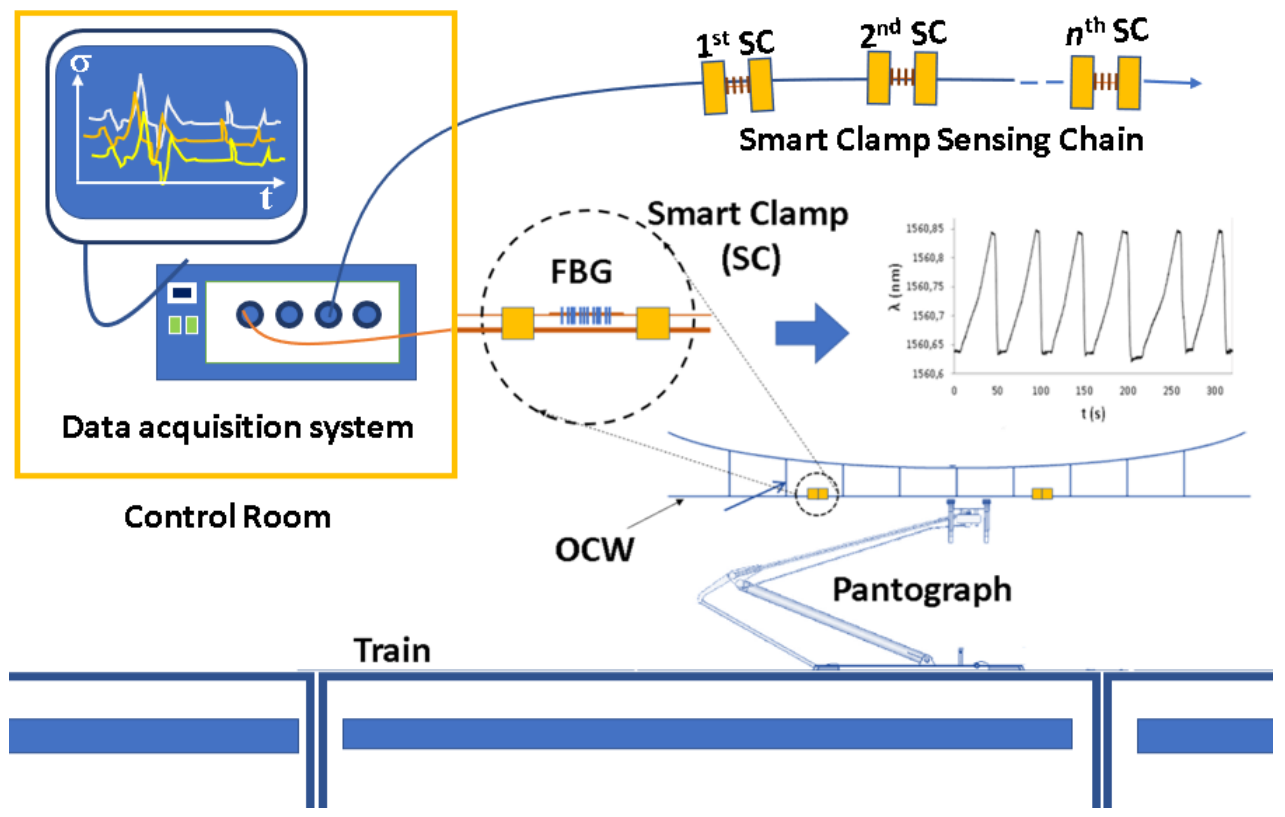

Figure 3: OCW Monitoring System Overview

This paper focuses attention on the sensitive part of this system, the OCW monitoring clamp. For that reason, an easy to install and remove system was designed, based on standard railway components and performing copper-coated FBG sensor (for allowing a safer handling and a better thermal and electrical conductivity). Two FBG-based clamps were developed, with two different geometries, two different materials and two FBG that differ in coating.

The paper is divided in two main sections, the first one describes the peculiarities that make the FBG the most suitable sensor for this application, the electrolytic process used to coat the optical fiber with a thin copper layer and a detailed description of the two clamps. The second section presents the experimental setup implemented to characterize the performance of the two clamps and summarizes the results of the tests. 


\section{MATERIALS AND METHODS}

$\mathrm{F}$ sensing system

iber optic sensors such as FBGs, [14], offer an excellent solution for being integrated into an OCW monitoring system for measuring strains, due to its unique features that are not commonly encountered in electrical monitoring systems. Among them, high strain and temperature sensitivity, electromagnetic immunity, minimum cabling, minimum weight and intrusiveness, and multiplex large number of sensors along the same (single) fiber capability and longdistance safe data acquisition are the most acknowledged. Furthermore, a feature that is very important for the aim of our research, is the possibility of coating the FBG by means of electrodeposition (ED) [15] with a thin layer of copper (Cu). In addition to mechanically reinforcing the fiber (and allowing their easier handling), the $\mathrm{Cu}$ coating increases the sensor thermal sensitivity [16]. Moreover, a metal coating makes FBGs more compatible for embedding within a metal matrix, such as in the case of OCS wires and of the Cu matrix of the clamps used as a gripping system in the Italian OCS. FBG sensors represent a technology, largely mature for measuring deformations in structures, as they are already used in many industrial applications, [17-20]. Fiber optic monitoring has been introduced in many engineering fields showing promising results even in the railways network field, providing very interesting performance while applied on smart railways projects [21, 22], or as an effective monitoring system for hard impacts on the OCW, in order to ensure efficient operation, [11]. Bragg gratings act as wavelength (WL) filters that are essentially defined by the microstructure spatial period and the refraction index of the fiber core [23]. The microstructure works as a WL selective mirror: light passing the fiber is partially backscattered and reflections are returned down in a narrow band. Max reflectivity occurs at the Bragg wavelength $\left(\lambda_{B}\right)$ and depends on the grating period, $\Lambda$, and on the effective index of refraction, $\mathrm{n}_{\mathrm{eff}}$, according to the Bragg condition:

$$
\lambda_{B}=2 n_{e f f} \Lambda
$$

The grating's WL changes with strain $(\varepsilon)$ and temperature $(T)$ according with:

$$
\frac{\Delta \lambda}{\lambda_{0}}=k \varepsilon+\alpha_{\delta} \Delta T
$$

Where $\Delta \lambda$ is the WL shift from the WL reference $\lambda_{0}$ ), $k$ is the grating gauge factor and $\alpha_{\delta}$ is the change in refraction index due to temperature change $(T)$. For the grating that have been used in this test campaign the $k$ is 0.89 . The sensing system applied on the OCW consists of optical fibers, integrated into the contact wire, along the existing connections, and an acquisition system that can be placed conveniently far from sensor locations. The fiber Bragg grating sensors are bonded directly on the clamps, as shown in Figs. 4 and 5, and the clamps are installed in selected places along the railways network.

\section{Copper coating electrodeposition on FBG sensor}

The FBGs that were used in the test campaign, were copper coated by means of the ED technique. The coating was performed to increase the fiber toughness resistance, the thermal conduction, to protect it from environmental conditions and to enhance the FBG sensitivity. Dummy (sensor-less fiber) samples were used for optimizing the ED process, while coated FBG samples (sensorized samples) were used for testing the proposed systems. The sample preparation (whether it is equipped with FBG or not) starts with the gilding of about $5 \mathrm{~cm}$ of fiber length by means of the sputtering technique (by using an EDWARDS sputter coating, model S150B) that was carried out in order to ensure the electrical conductivity of the outer surface (fused silica) of the fibers, that is necessary for the ensuing ED process. After the gold deposition, the cylindrical conductive surface is used as a cathode, while a custom-designed cylindrical lead anode is used to close the ED system. The metal ED was carried out by sinking the cathode-anode pair inside a glass electrolytic cell with radius and height of 3 and $12 \mathrm{~cm}$, respectively. The gilded fiber is kept on axis, at the center of the cylindrical cathode, and held vertically inside the cell, by means of a dedicated holder, in order to obtain a uniform thickness on the cylindrical surface, minimizing the occurrence of unwanted anisotropies or radial stresses in the coating. The presence of these defects can induce disturbances affecting the grating's spectrum. The maximum possible homogeneity of coating is required. The copper ED process was carried out using AMEL galvanostat and an electrolyte composition of $50 \mathrm{~g} / \mathrm{L} \mathrm{Cu}$ and $20 \mathrm{~g} / \mathrm{L} \mathrm{H}_{2} \mathrm{SO}_{4}$ by fixing the initial current density and temperature at $250 \mathrm{~A} / \mathrm{m}^{2}$ and $50{ }^{\circ} \mathrm{C}$, respectively. These ED process physical parameters affect: (i) thickness and (ii) grain size of the electrodeposited coating. The parameters values were selected by an optimization procedure performed on dummy samples, then used to produce the FBG samples. Finally, samples were examined by 
scanning electronic microscopy equipped with energy-dispersive X-ray spectroscopy (SEM-EDS) to determine the morphological aspect of the coatings.

\section{Smart Clamps}

For laboratory tests, two different clamp configurations were expressly designed and manufactured. The two configurations, called Smart Clamp 1 (SC1) and Smart Clamp 2 (SC2) differ in support geometry and in grating arrangement. The two SCs are shown in Figs. 4 and 5 and are described in detail in the following subsections. In developing the monitoring system, looking for a solution that was quickly removable, it was decided to use components off the shelf, i.e., components that were already used and approved by current legislation. Two different types of clamps already used in catenaries have been selected. A dropper jumpers clamp, that is made of copper, was used for SC1 and an electrical continuity jumpers clamp, that is in bronze, was used for SC2. Both items were instrumented with an FBG sensor, prepared with different coatings and arrangement. A method for installing sensors and preparing SCs was selected to allow easy mounting of the corresponding clamp, ensuring the standardization of the installation processes on the OCW. The FBG has been bonded on the external side of the clamps (closest as possible to the contact wire), in order to avoid its rupture possibly caused by the tightening torque of $25 \mathrm{Nm}$ that was used for installing the clamp on the contact wire.

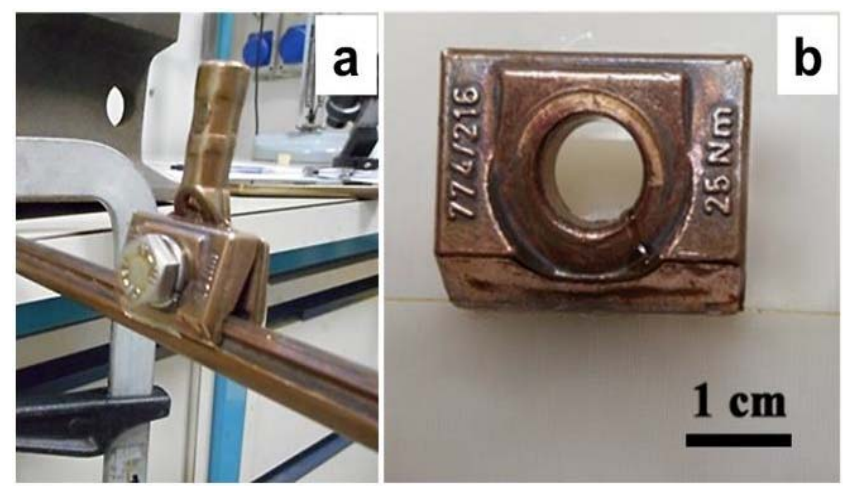

Figure 4: a) SC1; b) detail of the FBG bonded on SC1 copper clamp.

\section{SCs performance tests}

A mechanical tests campaign was performed on both SCs. It consists of three mechanical tests carried out to compare the performance of the two configurations. All tests were performed at room temperature (RT).

A first tensile test involves a single loading phase and was carried out to check the response of the two SCs to the mechanical tensile stress exerted on a OCW sample (a copper beam of $550 \mathrm{~mm}$ in length). The second test is a cyclic tensile test. In this case, the copper beam simulating the OCW was loaded and unloaded six times in a row to check the tightness of the SCs' bolts. Tensile tests simulate the system of counterweights that keeps the contact wire under tension. The maximum load imposed in these tests is $3 \mathrm{kN}$, that is the maximum target value of the Instron ${ }^{\circledR} 3375$ tensile testing machine that was used during these tests.

The copper alloys clamps are characterized by a high conductivity, therefore allowing a good detection of local temperature variations. Nevertheless, some preliminary qualitative observations were performed. A polyimide recoated FBG was used on the SC1, which was bonded using a two-component thermo-conductive epoxy glue. The sensor was bonded below the bolt to ensure maximum proximity to the OCW, Fig. 4.b. The Cu-coated sensor, described above, was installed on the SC2. In this case, the bronze clamp commonly used in electrical continuity jumpers was chosen. The original clamp was modified to reduce its stiffness, removing a central section, and keeping only the bolted ends, suspending the sensor between these two parts, as shown in Fig. 5. Thus, the mechanical stresses acting on the OCW (in particular those due to bending) are amplified. Furthermore, the Cu-coated grating being not in direct contact with the OCW, should be also slightly thermally isolated.

Furthermore, a bending test was performed on the setup shown in Fig. 6, to simulate the bending induced by the pantograph on the contact wire. The purpose of this simulation is to check the sensitivity of the two configurations in response to a minor mechanical stress, if compared with the pantograph motion, in accordance with the standard EN 50367:2006 that defines the range of application of the static force, between 60 and $70 \mathrm{~N}$, and the nominal value of the applied load, equal to $70 \mathrm{~N}$. The figure shows how the OCW sample, $550 \mathrm{~mm}$ long, has been suspended and fixed to the test-bench by means of a vice-clamp that prevents all its movement, blocking the beam's 6 degrees of freedom. The load was applied to the free 
end of the OCW while the SCs were mounted on it - with a tightening torque of $25 \mathrm{Nm}$ - at three successive positions from the joint, $z_{1}, z_{2}$ and $z_{3}$ as it is shown in Fig. 6. For both SCs the same load-conditions were applied (three different masses corresponding to $0.5 \mathrm{~kg}, 1 \mathrm{~kg}$ and $2 \mathrm{~kg}$ ). In all tests the load was applied and maintained in each position for 20 seconds, once the load was removed, the next load was applied after a 20 -second break.

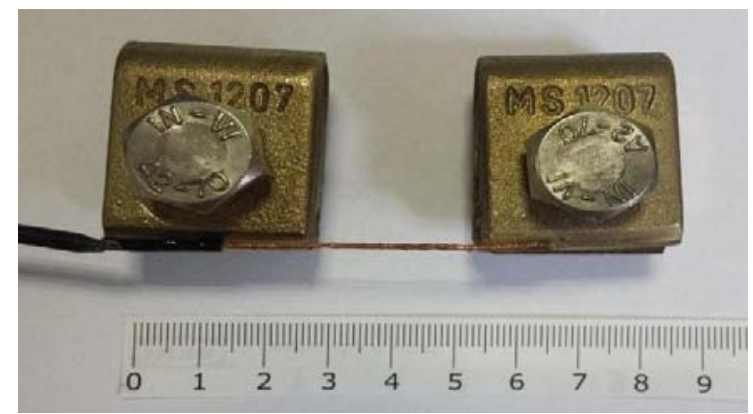

Figure 5: SC2 configuration, the grating is hanging between the two clamps.

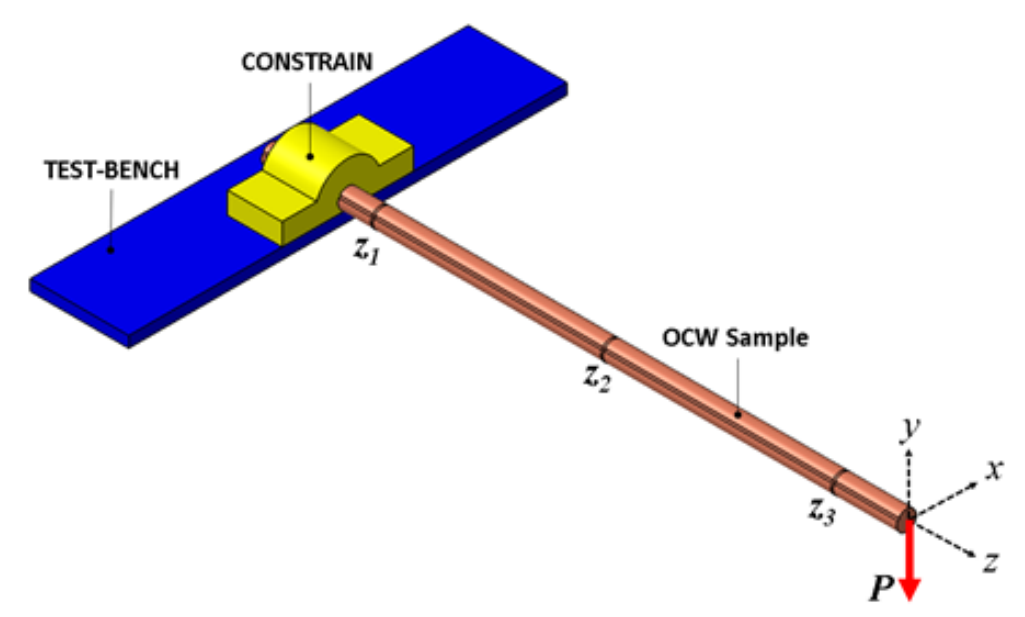

Figure 6: Bending test set-up.

This cantilever beam configuration represents a simplified model that allows to check the SCs sensitivity (i.e., their capability to measure even a smaller deformation induced on the OCW by a small load) and to compare the two SCs results with the calculated theoretical values. Simulating the wire as a cantilever beam, with well-known geometrical and mechanical characteristics, allows to effectively calculate the stress $(\sigma)$, through the typical equation:

$$
\sigma=\frac{M_{f}}{W_{f}}=M_{f} \frac{y_{\max }}{l_{x}}
$$

where $\mathrm{M}_{\mathrm{f}}$ is the bending moment and $\mathrm{W}_{\mathrm{f}}$ is the bending modulus $\left(y_{\max } / l_{x}\right.$, calculated as the ratio between the moment of inertia of the section - with regard to the neutral axis - and the furthest point of the section from the neutral axis). The strain ( $\varepsilon$ can be calculated by the equation that maintains its validity in the elastic field:

$$
\varepsilon=\frac{\sigma}{E}
$$

where $\mathrm{E}$ is the $\mathrm{OCW}$-alloy elastic modulus, that is $120 \mathrm{GPa}$ for the copper alloy considered during the tests, in compliance with national railway regulations. Since the strain decreases as the distance from the joint increases, different strain values were measured at the three different positions shown in Fig. $6\left(z_{1}, z_{2}\right.$ and $\left.z_{3}\right)$. 


\section{RESULTS AND DISCUSSION}

$\mathrm{T}$ his section presents and discusses the results concerning the ED of the copper coating on the optic fiber and how it affects the FBG's WL. Furthermore, the results of the test campaign that was carried out on the two SCs are presented.

\section{Sensor coating}

The dummy coated samples were used in destructive tests and SEM observations to evaluate the deposit quality in terms of both compactness and thickness uniformity on the cylindrical surface of the fiber. Fig. 7 provides several details of the dummy sample. The optical macrograph of a copper-coated sensor of Fig. 7a shows a good cylindrical shape, terminating in a truncated cone. That geometrical configuration indicates the conductive zone ending; Fig. 7b shows a complete view of the copper-coated sample appearing quite regular in its cylindrical form if considering the ED production process; Fig. $7 \mathrm{c}$ shows a sample cross section where in the right upper part a magnification of the sample emphasizes the gold thinner layer of about $3 \mu \mathrm{m}$. The cross-section shows a thick copper deposit of approximately $300 \mu \mathrm{m}$ with a consistent radius. Fig. $7 \mathrm{~d}$ highlights the dummy sample morphology where irregular and randomly disposed grains are evident, but the surface is compact and presents very few voids, as it is expected considering the copper positive deposition potential, that hinders the hydrogen evolution reaction (HER).

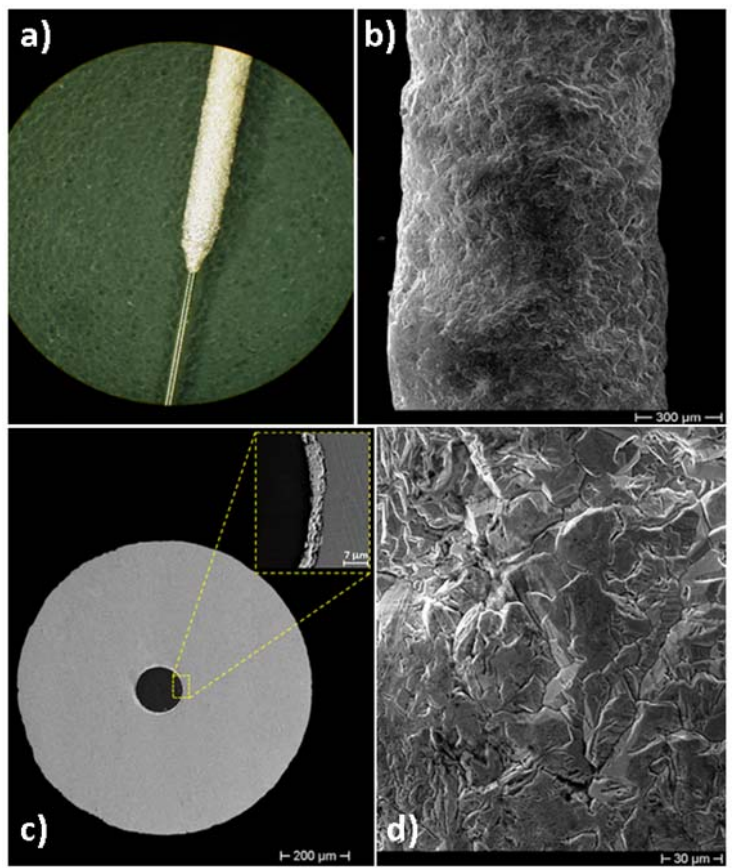

Figure 7: Optical macrographs and SEM micrographs showing geometrical and morphological aspects of the copper coated fiber: (a) optical general view, (b) SEM micrograph general view, (c) cross section and (d) surface morphology.

Fig. 8 shows the transformation of the grating spectrum following the various steps of sample coating production. After gilding (the blue line in Fig. 8), no relevant variations of the spectrum are revealed, while after $\mathrm{Cu}$ ED (the red line in Fig. 8), the spectrum experiences a broad shift toward lower wavelengths (WLs), with a small increase in intensity. The main effect of the thickness growth seems to be an appreciable WLs shift with quasi-unchanged spectral shape, that can be fully explained by a homogeneous axial contraction of the Bragg grating after the Cu ED.

\section{Tensile test}

The contact wire is usually tensioned by a system of counterweights in order to balance the deformations caused by temperature changes. Tensile tests were performed to simulate that operating conditions. The tests were carried out by mounting both SCs on the contact wire at the same time (Fig. 8) and associating a traditional extensometer to compare the maximum strain $\left(\varepsilon_{\max }\right)$ achieved during tests. 


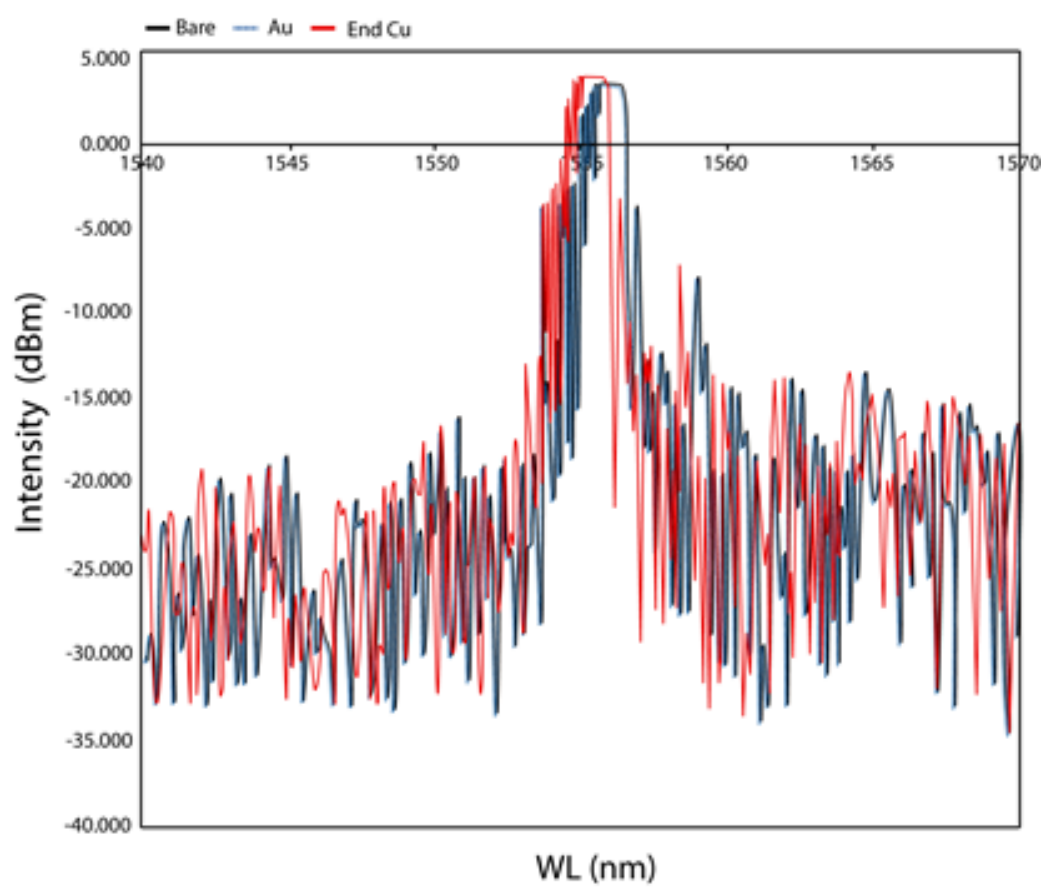

Figure 8: FBG spectrum shift due to the whole coating process.

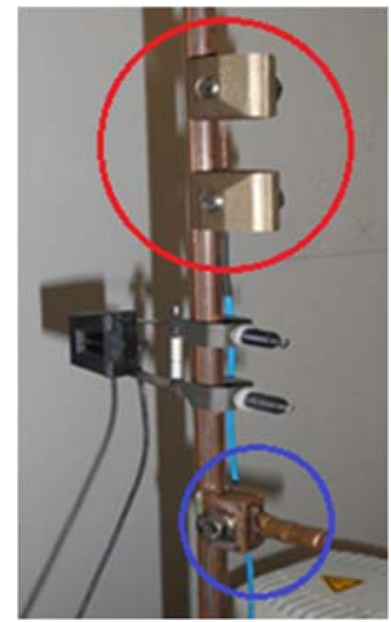

Figure 9: SC1(in blue) and SC2 (in red) mounted on the OCW segment undergoing tensile test.

The test setup (tensile test equipment and optical acquisition system) was set as follows:

- Imposed displacement: $0.5 \mathrm{~mm} / \mathrm{min}$

- Maximum applied load: $3 \mathrm{kN}$

- Contact wire length: $550 \mathrm{~mm}$

- Clamp tightening torque: $25 \mathrm{Nm}$

- Sampling frequency: $100 \mathrm{~Hz}$

The strain measured on SC1 and SC2 was recorded and reported in Fig. 10a and b, respectively. The two SCs measured different strains and, once again, the SC2 was more accurate. The WLs plots (Fig. 10a and b) show that SC2 has a higher sensitivity than SC1, being $\Delta \lambda$ around 0.2 and $0.01 \mathrm{~nm}$, respectively. The exact values for $\lambda_{0}, \lambda_{\text {Max }}, \Delta \lambda$, which are respectively the value at rest, the maximum reached value and the wavelength increment of the installed FBG, are reported in Tab. 1. The table also comes with the $\varepsilon_{\max }$ that is compared to the one obtained by tensile test machine's extensometer. The latest comparison shows that the SC2 strain and that measured by the extensometer are of the same order of magnitude while the 
SC1 measured strain is negligible. Fig. 10 highlights the latest observation, the sensor bonded on SC2 (Fig. 10b) records a maximum strain value 20 times higher than that measured by SC1 (Fig. 10a).

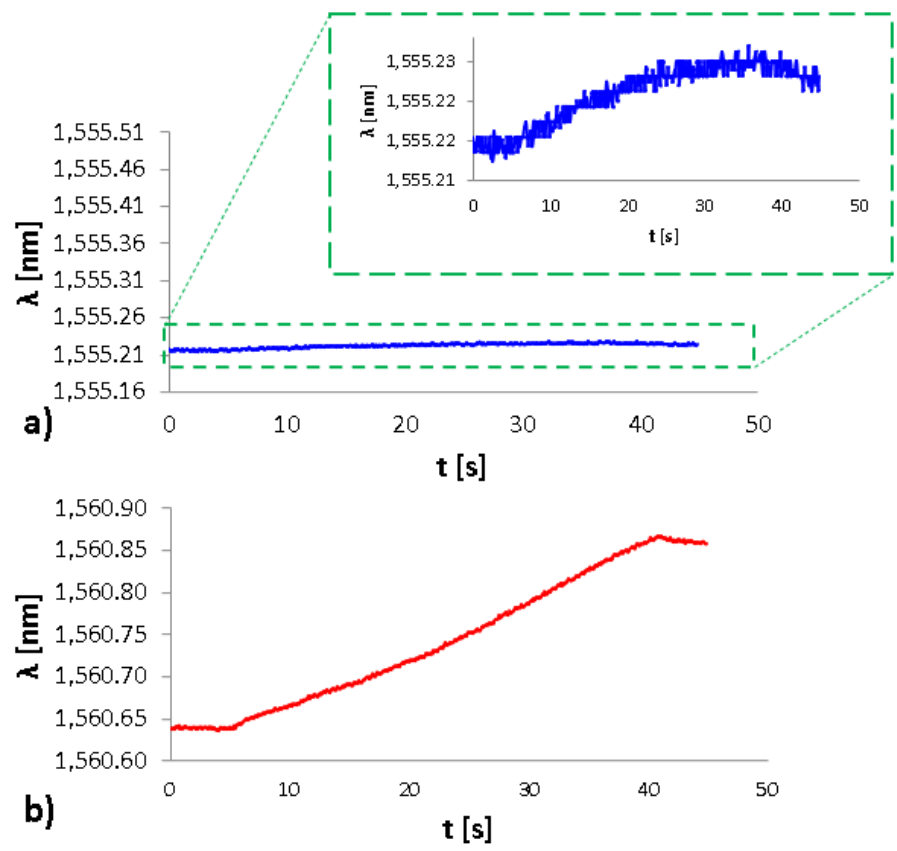

Figure 10: Tensile test on a) SC1 and on b) SC2. In a) the response profile of SC1 is highlighted even on a larger scale.

\begin{tabular}{lrrr}
\hline & SC 1 & SC 2 & Extensometer \\
$\lambda_{0}[\mathrm{~nm}]$ & 1555.216 & 1560.642 & - \\
$\lambda_{\text {Max Load }[\mathrm{nm}]}$ & 1555.227 & 1560.861 & - \\
$\Delta \lambda[\mathrm{nm}]$ & 0.011 & 0.220 & - \\
$\varepsilon_{\max }[\mu \mathrm{m} / \mathrm{m}]$ & 7.75 & 158.12 & 169.61 \\
\hline
\end{tabular}

Table 1: Tensile Test - Strain comparison.

\section{Cyclic tensile test}

A dynamic test was then carried out, using the same set-up and alternating 6 consecutive loading and unloading cycles, in order to verify the capability of the system to return to its starting (original) value in case of cyclic (repeated) stress. This cyclic tensile test is not a simulation of the real behavior of the OCW, which is subjected almost exclusively to bending, but rather a stress test carried out to check the tightness of the clamp bolts. The dynamic test confirms the previous observations and reveals an anomalous behavior of SC1, as shown in Fig. 11. The two SCs were tested simultaneously, yet the response to the same stress is different. The sensitivity of SC2 is visibly higher (Fig. 11b) than that of SC1 (Fig. 11a) and is emphasized by the same representation scale of $250 \mathrm{pm}$ of the vertical axis. The recorded WL variation is almost constant for both SCs but the sensitivity of SC2 is about 17 times higher than that of SC1. This ratio was calculated by considering the maximum WL increase reached by the two sensors at the maximum applied load $(3 \mathrm{kN})$. The strain results comparison of the cyclic tensile test is resumed in Tab. 2. The SC1 signal shows a marked trend of decreasing throughout the test, while SC2 remains stable. Based on these results, it can be assumed that the cause of the different behavior of the two clamps is due to a thermal effect. Although the two clamps are both mounted at the same time on the same OCW section during the cyclic test, their configuration induces a different response to a probable small local temperature variation. A local increase in temperature may have occurred during the test. Fig. 4 shows that the SC1 FBG is bonded directly onto the clamp (rigid and massive body), below the bolt, very close to the OCW. The SC2 sensor is hanging between two half clamps, in a position that is more distant from the OCW. It is also worth remembering that the thermal conductivity of copper is higher than that of bronze. These configurations make SC2 less responsive to temperature variations than SC1, which is more sensitive to thermal stress and less affected by mechanical stress. A second hypothesis could exclude temperature variation as a cause 
of the anomalous response of SC1 and instead could link it to the surface stress state induced by the very proximity of the bolt. In SC1 the grating is bonded very close to the bolt in an area that is probably under a compressive effect that makes unstable the perception of any other external mechanical stress.
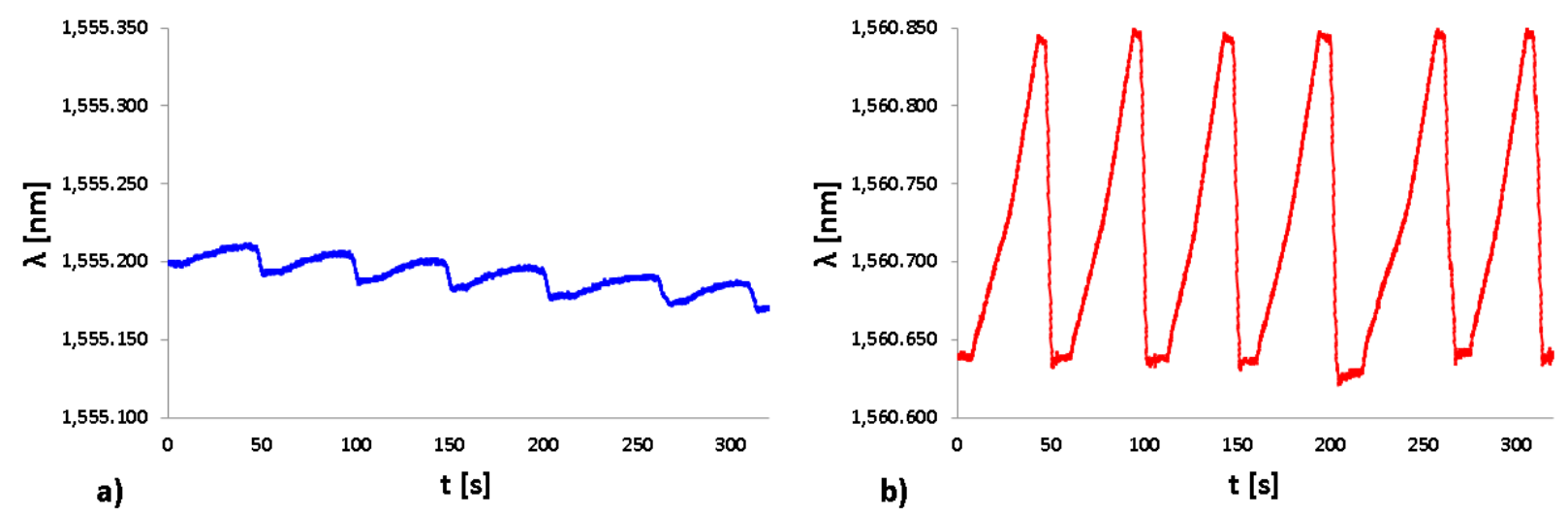

Figure 11: Cyclic tensile test on a) SC1 and b) SC2.

\begin{tabular}{lrrr}
\hline & FBG 1 & FBG 2 & Extensometer \\
$\lambda_{0}[\mathrm{~nm}]$ & 1555.1895 & 1560.637 & - \\
$\lambda_{\text {Max Load }}[\mathrm{nm}]$ & 1555.2012 & 1560.8468 & - \\
$\Delta \lambda[\mathrm{nm}]$ & 0.01165 & 0.19678 & - \\
$\varepsilon_{\max }[\mu \mathrm{m} / \mathrm{m}]$ & 8.42 & 151.03 & 167.44 \\
\hline
\end{tabular}

Table 2: Cyclic Tensile Test.

\section{Bending test}

The pantograph motion was simulated by means of a bending test, according to the setup shown in Fig. 6. This consists of a beam, made of an OCW segment, which was clamped at one end. The SCs were placed in the three positions indicated by z1, z2 and z3, which correspond to a distance of 30, 120 and $240 \mathrm{~mm}$ from the vise. In each test, a load (P) of 0.5 , 1.0 and $2.0 \mathrm{~kg}$ was hung at the free end of the beam. In this case the two SCs were not tested at the same time to ensure that they were positioned with the center of the grating at the indicated position.

The diagrams of Figs. 12, 13 and 14 show the WL variation recorded by the SC1 (plotted in blue) and SC2 (plotted in red) during the tests, with a sampling rate of $100 \mathrm{~Hz}$. Each graph was represented in a vertical range (WL) of $300 \mathrm{pm}$.

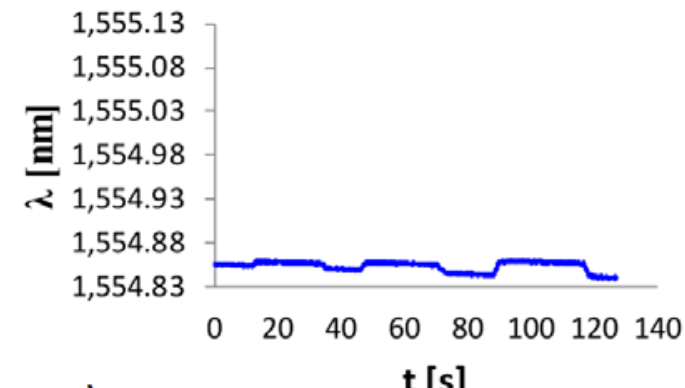

a)

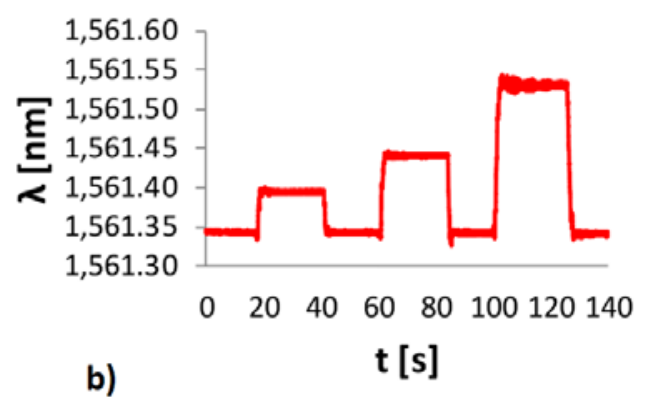

b)

Figure 12: Bending Test @z1 - sampling rate: 100 Hz. a) SC1 and b) SC2.

Tabs. 3 and 4 resumes the results collected during the bending tests, performed at $z_{1}, z_{2}$ and $z_{3}$ respectively on SC1 and SC2. The experimental values were compared with the values calculated by Eq. (3) e (4). 


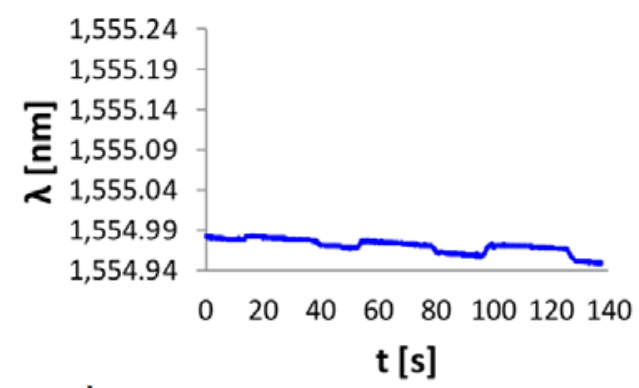

a)

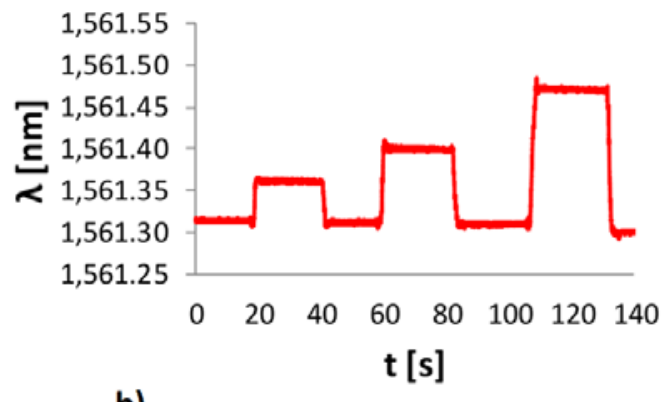

b)

Figure 13: Bending Test @zz - sampling rate: 100 Hz. a) SC1 and b) SC2.

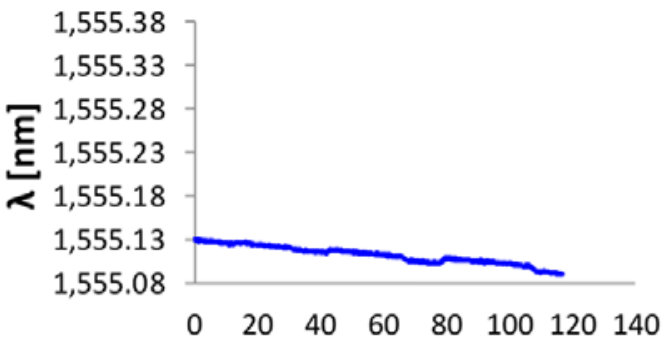

a) $\mathrm{t}$ [s]

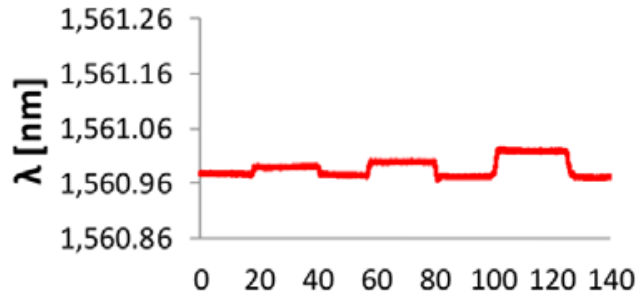

b)

$\mathrm{t}$ [s]

Figure 14: Bending Test@zz - sampling rate: 100 Hz. a) SC1 and b) SC2.

\begin{tabular}{|c|c|c|c|c|c|c|c|c|c|}
\hline \multirow[b]{2}{*}{ Applied Load [kg] } & \multicolumn{3}{|c|}{ z1 (30 mm) } & \multicolumn{3}{|c|}{$\mathrm{z} 2(120 \mathrm{~mm})$} & \multicolumn{3}{|c|}{ z3 (240 mm) } \\
\hline & 0.50 & 1.00 & 2.00 & 0.50 & 1.00 & 2.00 & 0.50 & 1.00 & 2.00 \\
\hline $\mathrm{M}_{\mathrm{f}}(\mathrm{z})[\mathrm{Nmm}]$ & 2650.00 & 5300.00 & 10600.00 & 2150.00 & 4300.00 & 8600.00 & 550.00 & 1100.00 & 2200.00 \\
\hline$\sigma(\mathrm{z})[\mathrm{MPa}]$ & 5.21 & 10.42 & 20.84 & 4.23 & 8.45 & 16.91 & 1.08 & 2.16 & 4.32 \\
\hline$\lambda_{0}[\mathrm{~nm}]$ & 1554.855 & 1554.851 & 1554.845 & 1554.977 & 1554.957 & 1554.958 & 1555.126 & 1555.116 & 1555.105 \\
\hline$\lambda_{\text {Load }}[\mathrm{nm}]$ & 1554.859 & 1554.857 & 1554.859 & 1554.982 & 1554.977 & 1554.971 & 1555.128 & 1555.12 & 1555.111 \\
\hline$\Delta \lambda[\mathrm{nm}]$ & 0.004 & 0.006 & 0.013 & 0.005 & 0.019 & 0.013 & 0.001 & 0.003 & 0.006 \\
\hline$\varepsilon[\mu \mathrm{m} / \mathrm{m}]$ & 43.41 & 86.83 & 173.65 & 35.22 & 70.44 & 140.89 & 9.01 & 18.02 & 36.04 \\
\hline$\varepsilon_{\mathrm{FBG}}[\mu \mathrm{m} / \mathrm{m}]$ & 2.75 & 4.22 & 9.62 & 2.29 & 4.12 & 5.96 & 1.00 & 2.40 & 4.15 \\
\hline$\varepsilon / \varepsilon_{\mathrm{FBG}}[\%]$ & 15.81 & 20.57 & 18.05 & 15.37 & 17.10 & 23.62 & 8.97 & 7.51 & 8.69 \\
\hline
\end{tabular}

Table 3: Bending Test Results on SC1.

\begin{tabular}{|c|c|c|c|c|c|c|c|c|c|}
\hline \multirow[b]{2}{*}{ Applied Load [kg] } & \multicolumn{3}{|c|}{ z1 (30 mm) } & \multicolumn{3}{|c|}{$\mathrm{z} 2(120 \mathrm{~mm})$} & \multicolumn{3}{|c|}{$\mathrm{z} 3(240 \mathrm{~mm})$} \\
\hline & 0.50 & 1.00 & 2.00 & 0.50 & 1.00 & 2.00 & 0.50 & 1.00 & 2.00 \\
\hline $\mathrm{M}_{\mathrm{f}}(\mathrm{z})[\mathrm{Nmm}]$ & 2600.00 & 5200.00 & 10400.00 & 1700.00 & 3400.00 & 6800.00 & 500.00 & 1000.00 & 2000.00 \\
\hline$\sigma(\mathrm{z})[\mathrm{MPa}]$ & 5.11 & 10.22 & 20.45 & 3.34 & 6.68 & 13.37 & 0.98 & 1.97 & 3.93 \\
\hline$\lambda_{0}[\mathrm{~nm}]$ & 1561.343 & 1561.343 & 1561.343 & 1561.314 & 1561.314 & 1561.314 & 1560.977 & 1560.977 & 1560.977 \\
\hline$\lambda_{\text {Load }}[\mathrm{nm}]$ & 1561.395 & 1561.441 & 1561.528 & 1561.362 & 1561.402 & 1561.471 & 1560.989 & 1561 & 1561.021 \\
\hline$\Delta \lambda[\mathrm{nm}]$ & 0.052 & 0.098 & 0.185 & 0.048 & 0.088 & 0.157 & 0.012 & 0.023 & 0.044 \\
\hline$\varepsilon[\mu \mathrm{m} / \mathrm{m}]$ & 42.59 & 85.19 & 170.38 & 27.85 & 55.70 & 111.40 & 8.19 & 16.38 & 32.76 \\
\hline$\varepsilon_{\mathrm{FBG}}[\mu \mathrm{m} / \mathrm{m}]$ & 37.34 & 70.44 & 133.03 & 34.50 & 63.51 & 113.30 & 8.94 & 16.55 & 31.73 \\
\hline$\varepsilon / \varepsilon_{\mathrm{FBG}}[\%]$ & 1.14 & 1.21 & 1.28 & 0.81 & 0.88 & 0.98 & 0.92 & 0.99 & 1.03 \\
\hline
\end{tabular}

Table 4: Bending Test Results on SC2. 
The last row of both tables shows the comparison between the strain recorded by the SCs and the theoretical (calculated) strain. SC2 recorded values very close to the calculated value. The bending tests confirm the findings of the tensile tests. SC2 shows a higher sensitivity to mechanical stress than SC1. Even in this case, the plot of the WLs of SC1 shows a barely perceptible reaction to the mechanical stress (i.e., load application at the free end of the beam), while it seems to react with a decreasing trend to a small local variation of the temperature. SC2, on the contrary, responds in a clear way to the application of the load and seems not to be influenced by the thermal stress.

\section{FINAL REMARKS}

$\mathrm{P}$ resent paper is aimed at seeking a smart solution for OCW continuous monitoring, a solution that meets the railway network requirements. The RFI demands a versatile sensor system capable of being easily installed (and removable) to existing networks, with no extra embedding procedures. This investigation proposes two easy to install and remove solutions (SC1 and SC2), both using the railways standard Cu-alloys clamps. The SC1 consists of a single copper element placed at the end of a dropper cable that hooks on the cable and remains in place by means of a single bolt; the sensor is an FBG coated only with polyimide coating and is bonded to the bottom of the clamp, very close to the OCW. The SC2 consists of two bronze half-clamps (obtained by cutting the original clamp in two parts) and a copper coated FBG sensor, which is hung between the two halves.

The operating conditions, simulated via bending and tensile tests, were evaluated using the SCs grabbed on an OCW used in the Italian RFI. The OCW was simulated using a copper (Cu- $0.1 \mathrm{wt} . \% \mathrm{Ag}$ alloy) beam segment of $55 \mathrm{~cm}$ in length, geometrically defined by the standard EN 50149/2013.

The strain measurements carried out by SC1 have shown unsatisfying results measuring strain values 20 times lower than that measured with SC2 (which recorded values that are very close to the calculated ones). Moreover, SC1 showed another issue concerning the difficulty of returning to its original WL. Plotted WL-time curve of both bending and tensile tests showed an anomalous descending trend. Two hypotheses have been proposed to understand this behavior, in both cases it should be recalled that the two SCs were tested under the same conditions and the response of SC2 was always consistent with the calculated values, responding linearly to the applied stresses and with a very low background noise. The copper coating plays a key role in the superior performance of the SC2. The electrodeposited Cu-coating confers greater rigidity to the grating which, combined with the configuration in which it is hanging between two half-clamps, allows the clamp to perform as a mechanical stress amplifier.

An in-depth metallographic analysis was performed on copper-coated optical fiber samples to check the good quality of the ED and to improve the deposition process.

\section{REFERENCES}

[1] Zhang, W., Zou, D., Tan, M., Zhou, N., Li, R., Mei, G. (2018). Review of pantograph and catenary interaction, Front. Mech. Eng., 13(2), pp. 311-22, DOI: 10.1007/s11465-018-0494-x.

[2] Cho, C.J., Park, Y. (2016). New Monitoring Technologies for Overhead Contact Line at $400 \mathrm{~km} \cdot \mathrm{h}-1$, Engineering, 2(3), pp. 360-365, DOI: 10.1016/J.ENG.2016.03.016.

[3] Antunes, P., Ambrósio, J., Pombo, J., Facchinetti, A. (2020). A new methodology to study the pantograph-catenary dynamics in curved railway tracks, Veh. Syst. Dyn., 58(3), pp. 425-452, DOI: 10.1080/00423114.2019.1583348.

[4] Shimanovsky, A., Yakubovich, V., Kapliuk, I. (2016). Modeling of the Pantograph-Catenary Wire Contact Interaction, Procedia Eng., 134, pp. 284-90, DOI: 10.1016/j.proeng.2016.01.009.

[5] Bucca, G., Collina, A. (2009). A procedure for the wear prediction of collector strip and contact wire in pantographcatenary system, Wear, 266(1-2), pp. 46-59, DOI: 10.1016/j.wear.2008.05.006.

[6] Mańka, A., Hełka, A., Ćwiek, J. (2020). Influence of Copper Content on Pantograph Contact Strip Material on Maximum Temperature of Railroad Wire, Sci. J. Silesian Univ. Technol. Ser. Transp., 106, pp. 97-105, DOI: $10.20858 /$ sjsutst.2020.106.8.

[7] Ambrósio, J., Pombo, J., Pereira, M. (2013). Optimization of high-speed railway pantographs for improving pantographcatenary contact, Theor. Appl. Mech. Lett., 3(1), pp. 013006, DOI: 10.1063/2.1301306.

[8] Borromeo, S., Aparicio, J.L., Martõâ, P.M. (2003). Proceedings of the Institution of Mechanical Engineers Part F, J. Rail Rapid Transit, 217(3), pp. 167-75, DOI: 10.1243/095440903769012876.

[9] Müller, R. (1997). Contact wire wear measurement devices. A system comparison. WCRR, vol. C, Firenze, pp. $245-50$. 
[10] Tan, M., Ning Zhou, Jiangwen Wang, Dong Zou, W.Z.\& G.M. (2018). A real-time impact detection and diagnosis system of catenary using measured strains by fibre Bragg grating sensors, Veh. Syst. Dyn., pp. 1-23, DOI: $10.1080 / 00423114.2018 .1556396$.

[11] EN 50125-1:2014. (2014). Railway applications. Environmental conditions for equipment. Rolling stock and on-board equipment.

[12] EN 50155:2017. (2017). Railway applications. Electronic equipment used on rolling stock.

[13] EN 50149:2012. (2012). Railway applications. Fixed installations. Electric traction. Copper and copper alloy grooved contact wires.

[14] Measures, R.M. (2001). Structural Monitoring with Fibre Optic Technology, Meas. Sci. Technol., 12(9), pp. 1609-10, DOI: $10.1088 / 0957-0233 / 12 / 9 / 708$.

[15] Lupi, C., Felli, F., Dell'Era, A., Ciro, E., Caponero, M.A., Kalinowski, H.J., Vendittozzi, C. (2019). Critical Issues of Double-Metal Layer Coating on FBG for Applications at High Temperatures, Sensors Artic., 19, pp. 3824, DOI: $10.3390 / \mathrm{s} 19183824$.

[16] Vendittozzi, C., Felli, F., Lupi, C. (2018). Modeling FBG sensors sensitivity from cryogenic temperatures to room temperature as a function of metal coating thickness, Opt. Fiber Technol., 42(February), pp. 84-91, DOI: 10.1016/j.yofte.2018.02.017.

[17] Ramzyzan Ramly, Wahyu Kuntjoro, M.K.A.R. (2012). Using Embedded Fiber Bragg Grating (FBG) Sensors in Smart Aircraft Structure Materials, Procedia Eng., 41, pp. 600-6, DOI: 10.1016/j.proeng.2012.07.218.

[18] Al-Fakih, E., Abu Osman, N.A., Mahamd Adikan, F.R. (2012). The Use of Fiber Bragg Grating Sensors in Biomechanics and Rehabilitation Applications: The State-of-the-Art and Ongoing Research Topics, Sensors, 12, pp. 12890-926, DOI: $10.3390 / \mathrm{s} 121012890$

[19] Regina C. S. B. Allil, Marcelo M. Werneck, B.A.R. and F.V.B. de N. (2013). Application of Fiber Bragg Grating Sensors in Power Industry, Current Trends in Short- and Long-period Fiber Gratings, IntechOpen.

[20] Allwood, G.; Wild, G.; Hinckley, S. (2017). Fiber Bragg Grating Sensors for Mainstream Industrial Processes, Electronics, 6(92), DOI: 10.3390/electronics6040092.

[21] Vendittozzi, C., Ciro, E., Felli, F., Lupi, C., Marra, F., Pulci, G., Astri, A. (2020). Static and dynamic weighing of rolling stocks by mean of a customized FBG-sensorized-patch, Int. J. Saf. Secur. Eng., 10(1), DOI: $10.18280 /$ ijsse.100111.

[22] Tam, Hwa \& Liu, Shun-Yee \& Guan, Bai-Ou \& Chung, Weng-Hong \& Chan, Tommy \& Cheng, L. (2005). Fiber Bragg Grating Sensors for Structural and Railway Applications. Proceedings of SPIE - The International Society for Optical Engineering.

[23] K. O. Hill and G. Meltz. (1997). Fiber Bragg grating technology fundamentals and overview, Light. Technol., 15(8), pp. 1263-76, DOI: 10.1109/50.618320. 